\title{
Some identities of higher-order Euler polynomials arising from Euler basis
}

\author{
by \\ Dae San Kim and Taekyun Kim
}

\begin{abstract}
The purpose of this paper is to present a systematic study of some families of higher-order Euler numbers and polynomials. In particular, by using the basis property of higher-order Euler polynomials for the space of polynomials of degree less than and equal to $n$, we derive some interesting identities for the higher-order Euler polynomials.
\end{abstract}

\section{Introduction}

As is well known, the $n$-th Euler polynomials of order $r$ are defined by the generating function to be

$$
\left(\frac{2}{e^{t}+1}\right)^{r} e^{x t}=e^{E^{(r)}(x) t}=\sum_{n=0}^{\infty} E_{n}^{(r)}(x) \frac{t^{n}}{n !} \quad\left(r \in \mathbf{Z}_{+}\right)
$$

with the usual convention about replacing $\left(E^{(r)}(x)\right)^{n}$ by $E_{n}^{(r)}(x)$ (see [1-11]). In the special case, $x=0, E_{n}^{(r)}(0)=E_{n}^{(r)}$ are called the $n$-th Euler numbers of order $r$.

By(1), we easily get

$$
\begin{aligned}
E_{n}^{(r)}(x) & =\sum_{l=0}^{n}\left(\begin{array}{c}
n \\
l
\end{array}\right) E_{l}^{(r)} x^{n-l}=\sum_{l=0}^{n}\left(\begin{array}{c}
n \\
l
\end{array}\right) E_{n-l}^{(r)} x^{l} \\
& =\sum_{n=n_{1}+\cdots+n_{r}+n_{r+1}}\left(\begin{array}{c}
n \\
n_{1}, \cdots, n_{r}, n_{r+1}
\end{array}\right) E_{n_{1}} E_{n_{2}} \cdots E_{n_{r}} x^{n_{r+1}} .
\end{aligned}
$$


From(2), we note that the leading coefficient of $E_{n}^{(r)}(x)$ is given by

$$
\sum_{n_{1}+\cdots+n_{r}=0}\left(\begin{array}{c}
n \\
n_{1}, \cdots, n_{r}
\end{array}\right) E_{n_{1}} E_{n_{2}} \cdots E_{n_{r}}=1
$$

Thus, $E_{n}^{(r)}(x)$ is a monic polynomial of degree $n$ with rational coefficients.

From(1), we have $E_{n}^{(0)}(x)=x^{n}$. It is not difficult to show that

$$
\frac{d E_{n}^{(r)}(x)}{d x}=n E_{n-1}^{(r)}(x), \quad E_{n}^{(r)}(x+1)+E_{n}^{(r)}(x)=2 E_{n}^{(r-1)}(x), \quad(\text { see [11-18]) }
$$

Now, we define two linear operators $\tilde{\triangle}$ and $D$ on the space of real-valued differentiable functions as follows:

$$
\tilde{\triangle} f(x)=f(x+1)+f(x), \quad D f(x)=\frac{d f(x)}{d x} .
$$

Then we see that $\tilde{\triangle} D=D \tilde{\triangle}$.

Let $V_{n}=\{p(x) \in \mathbf{Q}[x] \mid \operatorname{deg} p(x) \leq n\}$ be the $(n+1)$-dimensional vector space over Q. Probably, $\left\{1, x, \cdots, x^{n}\right\}$ is the most natural basis for $V_{n}$. But $\left\{E_{0}^{(r)}, E_{1}^{(r)}, \cdots, E_{n}^{(r)}\right\}$ is also a good basis for the space $V_{n}$ for our purpose of arithmetical and combinatorial applications of the higher-order Euler polynomials.

If $p(x) \in V_{n}$, then $p(x)$ can be expressed by

$$
p(x)=b_{0} E_{0}^{(r)}(x)+b_{1} E_{1}^{(r)}(x)+\cdots+b_{n} E_{n}^{(r)}(x) .
$$

In this paper, we develop methods for computing $b_{l}$ from the information of $p(x)$ and apply those results to arithmetically and combinatorially interesting identities involving $E_{0}^{(r)}, E_{1}^{(r)}, \cdots, E_{n}^{(r)}$.

\section{Higher-order Euler polynomials}

From(5), we have

$$
\tilde{\triangle} E_{n}^{(r)}(x)=E_{n}^{(r)}(x+1)+E_{n}^{(r)}(x)=2 E_{n}^{(r-1)}(x),
$$

and

$$
D E_{n}^{(r)}(x)=n E_{n-1}^{(r)}(x) .
$$


Let us assume that $p(x) \in V_{n}$. Then $p(x)$ can be generated by $E_{0}^{(r)}(x), E_{1}^{(r)}(x)$, $\cdots, E_{n}^{(r)}(x)$ to be

$$
p(x)=\sum_{k=0}^{n} b_{k} E_{k}^{(r)}(x) .
$$

Thus, by(18), we get

$$
\tilde{\triangle} p(x)=\sum_{k=0}^{n} b_{k} \tilde{\triangle} E_{k}^{(r)}(x)=2 \sum_{k=0}^{n} b_{k} E_{k}^{(r-1)}(x),
$$

and

$$
\tilde{\triangle}^{2} p(x)=2 \sum_{k=0}^{n} b_{k} \tilde{\triangle} E_{k}^{(r-1)}(x)=2^{2} \sum_{k=0}^{n} b_{k} E_{k}^{(r-2)}(x) .
$$

Continuing this process, we have

$$
\tilde{\triangle}^{r} p(x)=2^{r} \sum_{k=0}^{n} b_{k} E_{k}^{(0)}(x)=2^{r} \sum_{k=0}^{n} b_{k} x^{k} .
$$

Let us take the operator $D^{k}$ on (9). Then

$$
\begin{aligned}
D^{k} \tilde{\triangle}^{r} p(x) & =2^{r} \sum_{l=k}^{n} b_{l} l(l-1) \cdots(l-k+1) x^{l-k} \\
& =2^{r} \sum_{l=k}^{n} b_{l} \frac{l !}{(l-k) !} x^{l-k} \\
& =2^{r} \sum_{l=k}^{n} b_{l} k !\left(\begin{array}{c}
l \\
k
\end{array}\right) x^{l-k} .
\end{aligned}
$$

Let us take $x=0$ on (10). Then we get

$$
D^{k} \tilde{\triangle}^{r} p(0)=2^{r} b_{k} k ! .
$$

From (11), we have

$$
\begin{aligned}
b_{k} & =\frac{1}{2^{r} k !} D^{k} \tilde{\triangle}^{r} p(0)=\frac{1}{2^{r} k !} \tilde{\triangle}^{r} D^{k} p(0) \\
& =\frac{1}{2^{r} k !} \sum_{j=0}^{r}\left(\begin{array}{l}
r \\
j
\end{array}\right) D^{k} p(j) .
\end{aligned}
$$

Therefore, by (8) and(12), we obtain the following theorem. 
Theorem 1. For $n, r \in \mathbf{Z}_{+}, \quad p(x) \in V_{n}$, we have

$$
p(x)=\frac{1}{2^{r}} \sum_{k=0}^{n}\left(\sum_{j=0}^{r} \frac{1}{k !}\left(\begin{array}{c}
r \\
j
\end{array}\right) D^{k} p(j)\right) E_{k}^{(r)}(x) .
$$

Let us take $p(x)=x^{n} \in V_{n}$. Then we easily see that $D^{k} x^{n}=\frac{n !}{(n-k) !} x^{n-k}$. Thus, by Theorem 1, we get

$$
\begin{aligned}
x^{n} & =\frac{1}{2^{r}} \sum_{k=0}^{n} \sum_{j=0}^{r} \frac{1}{k !}\left(\begin{array}{c}
r \\
j
\end{array}\right) \frac{n !}{(n-k) !} j^{n-k} E_{k}^{(r)}(x) \\
& =\frac{1}{2^{r}} \sum_{k=0}^{n} \sum_{j=0}^{r}\left(\begin{array}{c}
r \\
j
\end{array}\right)\left(\begin{array}{c}
n \\
k
\end{array}\right) j^{n-k} E_{k}^{(r)}(x) .
\end{aligned}
$$

Therefore, by (13), we obtain the following corollary.

Corollary 2. For $n, r \in \mathbf{Z}_{+}$, we have

$$
x^{n}=\frac{1}{2^{r}} \sum_{k=0}^{n} \sum_{j=0}^{r}\left(\begin{array}{c}
r \\
j
\end{array}\right)\left(\begin{array}{l}
n \\
k
\end{array}\right) j^{n-k} E_{k}^{(r)}(x) .
$$

Let $p(x)=B_{n}^{(s)}(x)\left(s \in \mathbf{Z}_{+}\right)$. Then we have

$$
D^{k} B_{n}^{(s)}(x)=\frac{n !}{(n-k) !} B_{n-k}^{(s)}(x) .
$$

By Theorem 1, we get

$$
B_{n}^{(s)}(x)=\frac{1}{2^{r}} \sum_{k=0}^{n} \sum_{j=0}^{r}\left(\begin{array}{c}
r \\
j
\end{array}\right)\left(\begin{array}{c}
n \\
k
\end{array}\right) B_{n-k}^{(s)}(j) E_{k}^{(r)}(x) .
$$

Therefore, by (15), we obtain the following corollary.

Corollary 3. For $n, s, r \in \mathbf{Z}_{+}$, we have

$$
B_{n}^{(s)}(x)=\frac{1}{2^{r}} \sum_{k=0}^{n} \sum_{j=0}^{r}\left(\begin{array}{c}
r \\
j
\end{array}\right)\left(\begin{array}{c}
n \\
k
\end{array}\right) B_{n-k}^{(s)}(j) E_{k}^{(r)}(x),
$$

where $B_{n}^{(s)}(x)$ are the $n$-th Bernoulli polynomials of order $s$. 
It is well known that

$$
\frac{t}{e^{t}-1} e^{x t}=\sum_{n=0}^{\infty} B_{n}(x) \frac{t^{n}}{n !}, \quad \frac{2}{e^{t}+1} e^{x t}=\sum_{n=0}^{\infty} E_{n}(x) \frac{x^{n}}{n !} .
$$

In the special case, $x=0$, let $B_{n}(0)=B_{n}, E_{n}(0)=E_{n}$. From(16) , we easily derive the following identity:

$$
B_{n}(x)=\sum_{k=0, k \neq 1}^{n}\left(\begin{array}{c}
n \\
k
\end{array}\right) B_{k} E_{n-k}(x) \in V_{n} .
$$

Let us take $p(x)=B_{n}(x)$. Then we have

$$
D^{k} B_{n}(x)=n(n-1) \cdots(n-k+1) B_{n-k}(x)=\frac{n !}{(n-k) !} B_{n-k}(x) .
$$

Therefore, by Theorem 1, (17) and (18), we obtain the following theorem.

Theorem 4. For $n, r \in \mathbf{Z}_{+}$, we have

$$
\sum_{k=0, k \neq 1}^{n}\left(\begin{array}{c}
n \\
k
\end{array}\right) B_{k} E_{n-k}(x)=\frac{1}{2^{r}} \sum_{k=0}^{n} \sum_{j=0}^{r}\left(\begin{array}{l}
r \\
j
\end{array}\right)\left(\begin{array}{l}
n \\
k
\end{array}\right) B_{n-k}(j) E_{k}^{(r)}(x) .
$$

Let us consider $p(x)=\sum_{k=0}^{n} B_{k}(x) B_{n-k}(x)$.

Then we have

$$
D^{k} p(x)=\frac{(n+1) !}{(n-k+1) !} \sum_{l=k}^{n} B_{l-k}(x) B_{n-l}(x) .
$$

Thus, by Theorem 1 and (19), we obtain the following theorem.

Theorem 5. For $r, n \in \mathbf{Z}_{+}$, we have

$$
\sum_{k=0}^{n} B_{k}(x) B_{n-k}(x)=\frac{1}{2^{r}} \sum_{k=0}^{n} \sum_{l=k}^{n} \sum_{j=0}^{r}\left(\begin{array}{c}
r \\
j
\end{array}\right)\left(\begin{array}{c}
n+1 \\
k
\end{array}\right) B_{l-k}(j) B_{n-l}(j) E_{k}^{(r)}(x) .
$$


Let $n, m \in \mathbf{Z}_{+}$, with $n \geq m+2$. Then we have

$$
\begin{aligned}
& B_{m}(x) B_{n-m}(x) \\
& =\sum_{l=0}^{\infty}\left\{\left(\begin{array}{c}
m \\
2 l
\end{array}\right)(n-m)+\left(\begin{array}{c}
n-m \\
2 l
\end{array}\right) m\right\} \frac{B_{2 l} B_{n-2 l}(x)}{n-2 l}+(-1)^{m+1} \frac{B_{n}}{\left(\begin{array}{c}
n \\
m
\end{array}\right)} .
\end{aligned}
$$

Let us take $p(x)=B_{m}(x) B_{n-m}(x) \in V_{n}$.

Then we have

$$
\begin{aligned}
& D^{k} p(x) \\
& =\sum_{l=k}^{\infty}\left\{\left(\begin{array}{l}
m \\
2 l
\end{array}\right)(n-m)+\left(\begin{array}{c}
n-m \\
2 l
\end{array}\right) m\right\} \frac{B_{2 l}}{n-2 l} \times \frac{(n-2 l) !}{(n-2 l-k) !} B_{n-2 l-k}(x) .
\end{aligned}
$$

Therefore, by Theorem 1 and (21), we obtain the following theorem.

Theorem 6. For $n, m \in \mathbf{Z}_{+}$with $n \geq m+2$, we have

$$
\begin{aligned}
& B_{m}(x) B_{n-m}(x) \\
& =\frac{1}{2^{r}} \sum_{k=0}^{n}\left\{\sum_{l=k}^{\infty} \sum_{j=0}^{r}\left(\begin{array}{c}
r \\
j
\end{array}\right)\left(\begin{array}{c}
n-2 l \\
k
\end{array}\right)\right. \\
& \left.\times\left(\left(\begin{array}{c}
m \\
2 l
\end{array}\right)(n-m)+\left(\begin{array}{c}
n-m \\
2 l
\end{array}\right) m\right) \frac{B_{2 l} B_{n-2 l-k}(j)}{n-2 l}\right\} E_{k}^{(r)}(x) .
\end{aligned}
$$

Remark. By using Theorem 1, we can find many interesting identities related to Bernoulli and Euler polynomials. 


\section{References}

[1] M.Abramowitz, I. A. Stegun, Handbook of Mathematical Functions, National Bureau of Standards, Washington, DC, 1964.

[2] S. Araci, D. Erdal, Higher order Genocchi, Euler polynomials associated with q-Bernstein type polynomials, Honam Math. J. 33(2011), no. 2, 173179.

[3] A. Bayad, T. Kim, Identities involving values of Bernstein, q-Bernoulli, and q-Euler polynmials, Russ. J. Math. Phys. 18(2011), no.2, 133-143.

[4] M. Cenkci, Y. Simsek, V. Kurt, Multiple two-variable p-adic q-L-function and its behavior at $s=0$, Russ. J. Math. Phys. 15(2008), no. 4, 447-459.

[5] H.W. Gould, Explicit formulas for Bernoulli numbers, Amer. Math. Monthly 79(1972), 44-51.

[6] L.-C. Jang, A study on the distribution of twisted q-Genocchi polynomials, Adv. Stud. Contemp. Math. 18 (2009), no. 2, 181-189.

[7] D. S. Kim, T. Kim, Euler basis, identities, and their applications, Int. J. Math. Math. Sci., 2012(2012), Article ID 343981, 15 pages. doi:10.1155/2012/343981

[8] D. S. Kim, T. Kim, Bernoulli basis and the product of several Bernoulli polynomials, Int. J. Math. Math. Sci., 2012(2012), Article ID 463659, 12pages. doi:10.1155/2012/463659

[9] T. Kim, Sums of products of q-Bernoulli numbers, Arch. Math. (Basel) 76(2001), no. 3, 190-195.

[10] T. Kim, C. Adiga, Sums of products of generalized Bernoulli numbers, Int. Math. J. 5(2004), no. 1, 1-7.

[11] T. Machide, Sums of products of Kronecker's double series, J. Number Theory 128(2008), no. 4, 820-834.

[12] H. Ozden, p-adic distribution of the unification of the Bernoulli, Euler and Genocchi polynomials, Appl. Math. Comput. 218(2011), no. 3, 970973.

[13] H. Ozden, Y. Simsek, S.-H. Rim, I. N. Cangul, A note on p-adic q-Euler measure, Adv. Stud. Contemp. Math. 14(2007), no. 2, 223-239. 
[14] A. Petojević, New sums of products of Bernoulli numbers, Integral Transforms Spec. Funct. 19(2008), no.1-2, 105-114.

[15] S.-H. Rim, J. Jeong, On the modified q-Euler numbers of higher order with weight, Adv. Stud. Contemp. Math. 22(2012), no. 1, 93-98.

[16] C. S. Ryoo, Some relations between twisted q-Euler numbers and Bernstein polynomials, Adv. Stud. Contemp. Math. 21(2011), no. 2, 217-223.

[17] Y. Simsek, Complete sum of products of $(h, q)$-extension of Euler polynomials and numbers, J. Difference Equ. Appl. 16(2010), no. 11, 1331-1348.

[18] Y. Simsek, Special functions related to Dedekind-type DC-sums and their applications, Russ. J. Math. Phys. 17 (2010), no. 4, 495-508.

Department of Mathematics, Sogang University, Seoul 121-742, Republic of Korea

e-mail: dskim@sogang.ac.kr

Department of Mathematics, Kwangwoon University, Seoul 139-701, Republic of Korea

e-mail: tkkim@kw.ac.kr 